\title{
Cilioretinal Artery Hypoperfusion in Association with Paracentral Acute Middle Maculopathy: A Case Report and Review of Literature
}

\author{
Elias Khalili Pour, Hamid Riazi Esfahani, Shahin Faghihi and Hooshang Faghihi* \\ Eye research center, Farabi Eye Hospital, Iran \\ *Corresponding author: Hooshang Faghihi, Assistant professor of ophthalmology , Vitreoretinal Fellowship Eye Research \\ Center, Farabi Eye Hospital, Iran
}

\section{ARTICLE INFO

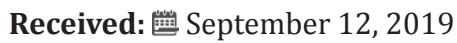 \\ Published: 慧 September 19, 2019}

Citation: Elias Khalili Pour, Hamid Riazi Esfahani, Shahin Faghihi, Hooshang Faghihi. Cilioretinal Artery Hypoperfusion in Association with Paracentral Acute Middle Maculopathy: A Case Report and Review of Literature. Biomed J Sci \& Tech Res 21(3)-2019. BJSTR. MS.ID.003604.

\section{ABSTRACT}

We report a case of unilateral cilioretinal artery hypoperfusion with 20/20 visual acuity accompanied by paracentral acute middle maculopathy in the marginal zones of the ischemic area. Multimodal imaging of the patient including fundus autofluorescence, spectral-domain optical coherence tomography (SD-OCT) and optical coherence tomography angiography (OCTA) was done. In this case, we demonstrated the most noteworthy susceptibility of the retina to hypoperfusion at the level of the deep capillary plexus.

Abbreviations: SD-OCT: Spectral-Domain Optical Coherence Tomography; OCTA: Optical Coherence Tomography Angiography; PAMW: Paracentral Acute Middle Maculopathy; PDE-5: Phosphodiesterase-5; PAMM: Paracentral Acute Middle Maculopathy

\section{Introduction}

Paracentral acute middle maculopathy (PAMM) has been described as an independent entity or accompanied by other retinal vascular diseases like diabetic retinopathy, retinal vein occlusion, retinal artery occlusion and with the consumption of vasoconstrictors like caffeine and epinephrine [1,2]. In this report we present a case of cilioretinal artery hypoperfusion and PAMM in the border of the affected ischemic retinal area and will review the literature that showed the vulnerability of deep capillary plexus to the ischemic conditions.

\section{Case Report}

A 47- year-old man was referred to retina ward of Farabi eye hospital with the complaint of scotoma in front of the right eye since 2 days ago upon awakening in the morning. He was a nonsmoker diabetic patient under treatment with Metformin. $\mathrm{He}$ was hypertensive and with consumption of 50 milligrams daily Losartan, blood pressure was under control. He did not suffer from migraine and other types of headaches, recent traumatic history, abnormal caffeine intake, phosphodiesterase-5 (PDE-5) inhibitor consumption at bedtime and so on.

Best-corrected visual acuity was $20 / 20$ in both eyes. Anterior segment examination was unremarkable. Fundus evaluation of the right eye showed a yellow-white well-defined lesion in the distribution of the cilioretinal artery (Figures 1A \& 1B). Left eye fundus evaluation was normal (Figure 1C). SD-OCT through the lesion (Figures 1D \& 1E) demonstrated a placoid, hyperreflective band at the level of the inner and middle retinal layers consistent with cilioretinal artery hypoperfusion and at the lower border of the lesion this hyperreflective band was just at the level of middle retina (inner nuclear layer) consistent with paracentral acute middle maculopathy with extension into the inner plexiform layer. Fundus autofluorescence showed hypo autofluorescence at the level of lesion (Figure 1F). OCT Angiography with En face projection (Figure 1G) at the level of the deep capillary plexus showed a gross capillary loss with visibility of superficial retinal vessels seen as projection artifacts. OCTA of the left eye was normal (Figure $1 \mathrm{H}$ ). 


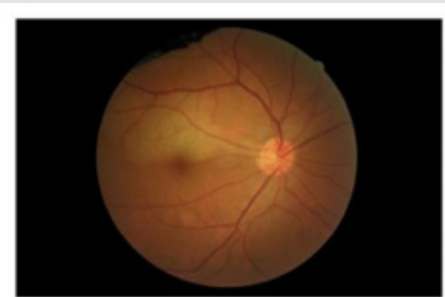

A
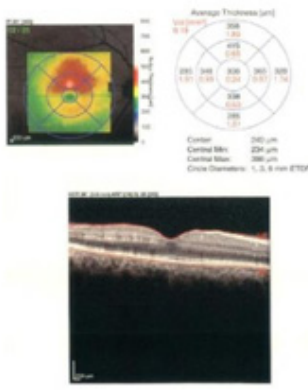
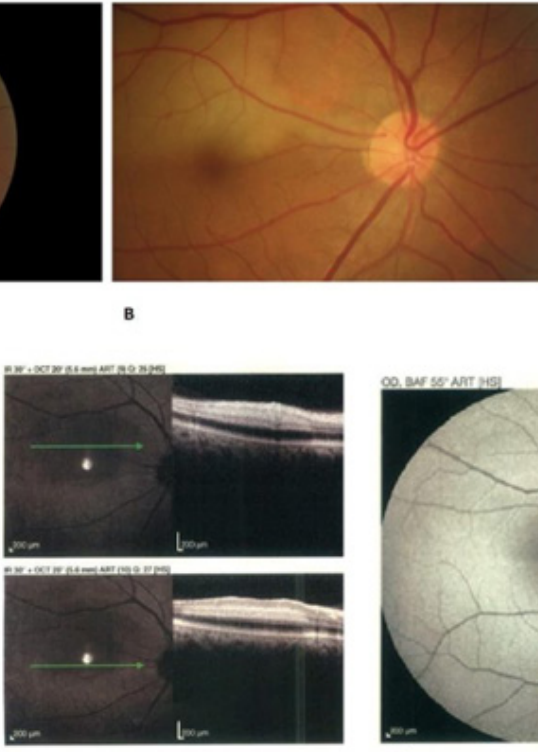

E
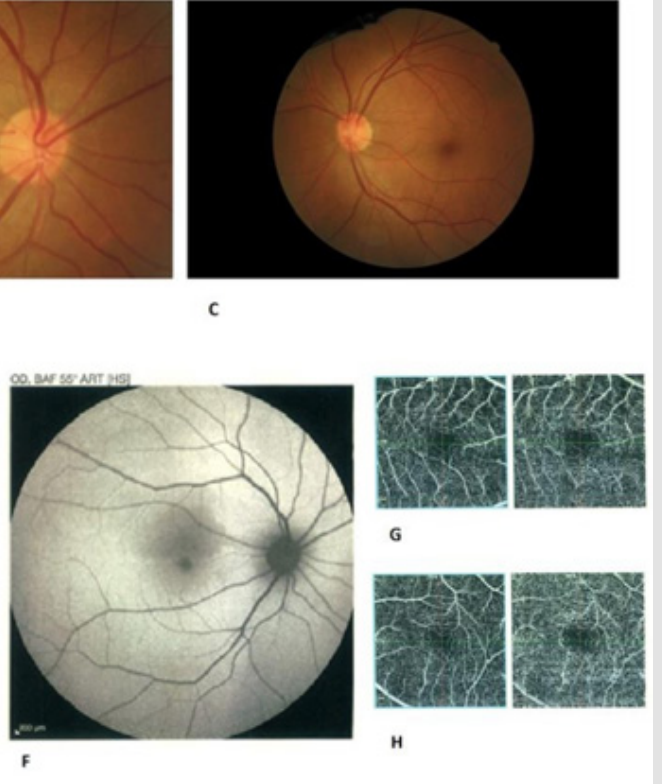

Figure 1:

A. Fundus photograph of the right eye two days after initiation of symptoms. Yellow white well-defined lesion in the distribution of the cilioretinal artery and just above the fovea can be seen.

B. Magnified view of the right eye fundus shows the cilioretinal artery that exits from the optic disc.

C. Normal left eye fundus examination.

D. Optical coherence tomography through the fovea and just at the lower border of retinal yellow-white lesion demonstrates hyperreflective bands in a skip pattern at the level of middle retinal layers with extension into the inner plexiform layer consistent with paracentral acute middle maculopathy (PAAM).

E. Placoid, hyperreflective band at the level of the inner and middle retinal layers consistent with cilioretinal artery hypoperfusion.

F. Fundus autofluorescence showed hypo autofluorescence at the level of lesion.

G. OCT Angiography with En face projection at the level of the deep capillary plexus showed a gross capillary loss with visibility of superficial retinal vessels seen as projection artifacts.

H. OCTA of the left eye was normal.

\section{Discussion}

In this case, we reported a middle-aged man with 20/20 BCVA and a scotoma in front of the right eye and acute retinal whitening consistent with the diagnosis of hypoperfusion in the distribution of cilioretinal artery and PAMM at the lower border of whitening site. The central retinal artery and cilioretinal artery arise from different vascular frameworks and have distinctive physiological properties [3]. As opposed to the central retinal artery, the cilioretinal artery arises from the peripapillary choroid or directly from one of the short posterior ciliary arteries and isn't liable to autoregulation. Subsequently, a drop in systemic blood pressure may cause a secondary reduction in the perfusion pressure and relative hypoperfusion or insufficiency of the cilioretinal artery $[4,5]$.

A critical decrease of blood pressure can occur physiologically during sleep and may assume a significant role in the pathogenesis and evolution of isolated cilioretinal artery occlusion. In this case, we showed cilioretinal artery hypoperfusion accompanying paracentral acute middle maculopathy (PAMM) in the border of the low-perfusion area as a hyperreflective band in the inner nuclear layer in SD-OCT images. Due to relatively preserved vision of this patient and very mild edema in the inner retinal layers in the affected area (unlike the retinal artery occlusion cases that inner retina has severe ischemic whitening), it is better to say cilioretinal artery, in this case, has hypoperfusion not occlusion although fluorescein angiography can prove this claim. Recent studies showed that deep capillary plexus of the retina is the watershed zone of inner retinal blood supply and receives oxygenated blood from superficial capillary plexus (Figure 2A-2D). So, any disturbance in the perfusion of the retina like nocturnal hypotension can first affect inner capillary plexus. In this case we can see that regions of retina that are located near the central zones of the cilioretinal artery hypoperfusion are more ischemic than marginal zones and have edema in both inner and middle retinal layers like cases of branch retinal artery occlusion although inner retina has mild edema due to insufficiency not occlusion of cilioretinal artery, but as we go farther to the margin of the affected area we can see that the only middle retinal layers (inner nuclear layer) are affected and PAAM in these areas has a skip pattern and inner retinal layers like RNFL look normal. This pattern can be described by this fact that low perfusion in these marginal zones predominantly affect inner capillary plexus as a watershed zone and so just middle retinal layers shows hyperreflectivity in OCT. On the other hand, 
superficial retinal layers of the marginal zones of the affected area can be supplied by other arterioles around the ischemic area but middle retinal layers like inner nuclear layer are still more ischemic than superficial layers due to dependency of middle layers to superficial layers (Figure 2). This case presents a simplified model of the ischemic cascade that introduced by Sarraf et al. (2018) [6] \& (Figure 2).

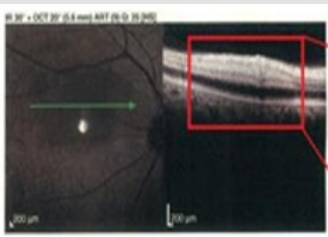

A

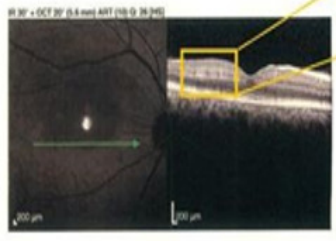

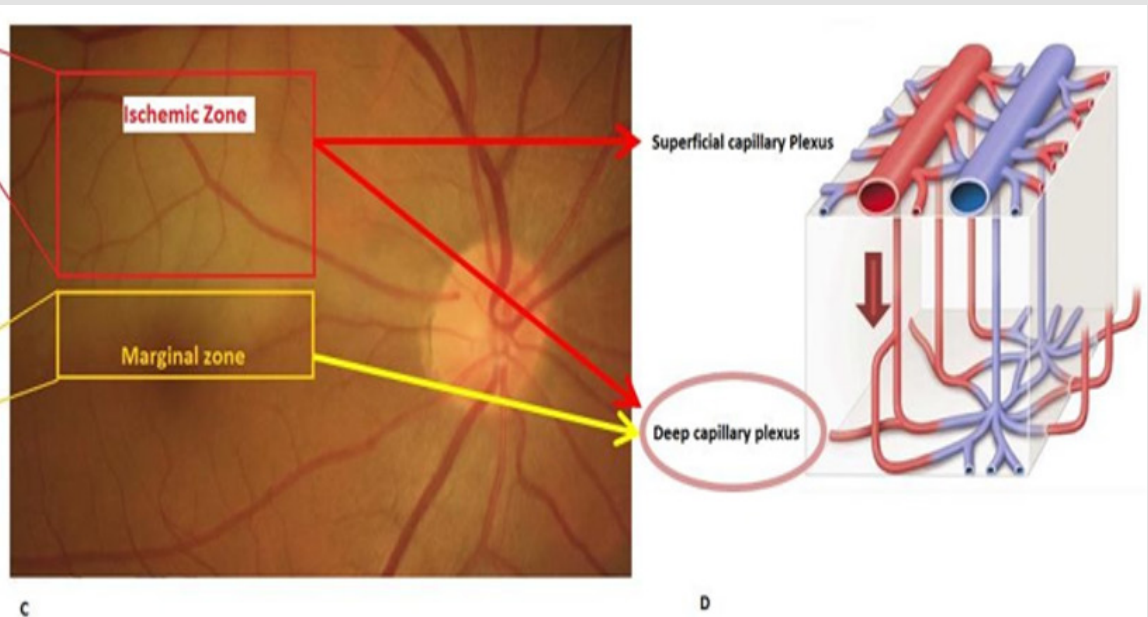

Figure 2: SD-OCT of the ischemic zone (A) around the cilioretinal artery hypoperfusion area ( red rectangle in fundus photo (C)) and SD-OCT of marginal zone (B) and corresponding fundus photo (yellow rectangle in C) and schematic view of superficial and deep capillary plexus of the retina. In the ischemic zone both superficial and deep capillary plexuses are affected (red arrows) but in the marginal zone just deep capillary plexus in affected (red arrow).

In this case, we demonstrated the most noteworthy susceptibility of the retina to hypoperfusion at the level of the DCP. This finding has been recently watched and might be clarified by the nearness of higher oxygen levels or better collateralization at the marginal regions of the infarct contiguous to normal retinal tissue $[7,8]$. Besides the conditions like retinal artery occlusion or insufficiency that make the deep capillary plexus susceptible to hypoperfusion and resulting PAAM in this area, this plexus is also vulnerable to conditions like retinal vein occlusion and PAAM can be seen in conditions affecting venous drainage of the retina. Recent enface OCT imaging and OCTA studies have shown that micro vortex-vein like structures are available in the deep capillary plexus and these structures are responsible for the drainage of the middle retinal layers, so any occlusion in front of the venous pathway can predominantly affect this area and secondary PAAM can be seen here $[4-6,8]$. So, it seems that deep capillary plexus is a sensitive structure for both arterial and venous insufficiencies and multimodal imaging studies can show this vulnerability to vascular disturbances.

\section{References}

1. Roy R, Shah D, Saurabh K (2018) Multimodal imaging in paracentral acute middle maculopathy. Indian Journal of Ophthalmology 66(8): 1186-1188.
2. Tsui I, Sarraf D (2013) Paracentral Acute Middle Maculopathy and Acute Macular Neuroretinopathy. Ophthalmic Surgery, Lasers, and Imaging Retina 44(6): 33-35.

3. Hayreh SS (2011) Acute retinal arterial occlusive disorders. Prog Retin Eye Res 30(5): 359-394.

4. Pichi F, Fragiotta S, Freund KB, Au A, Lembo A, et al. (2019) Cilioretinal artery hypoperfusion and its association with paracentral acute middle maculopathy. Br J Ophthalmol 103(8): 1137-1145.

5. Rahimy E, Sarraf D (2014) Paracentral acute middle maculopathy spectral-domain optical coherence tomography feature of deep capillary ischemia. Current Opinion in Ophthalmology 25(3): 207-212.

6. Bakhoum MF, Freund KB, Dolz Marco R, Leong BCS, Baumal CR, et al. (2018) Paracentral Acute Middle Maculopathy and the Ischemic Cascade Associated With Retinal Vascular Occlusion. Am J Ophthalmol 195: 143153.

7. Yu S, Pang CE, Gong Y, Freund KB, Yannuzzi LA, et al. (2015) The spectrum of superficial and deep capillary ischemia in retinal artery occlusion. Am J Ophthalmol 159(1): 53-63.

8. Ghasemi Falavarjani K, Phasukkijwatana N, Freund KB, Cunningham ET, Kalevar A, et al. (2017) En Face Optical Coherence Tomography Analysis to Assess the Spectrum of Perivenular Ischemia and Paracentral Acute Middle Maculopathy in Retinal Vein Occlusion. Am J Ophthalmol 177: 131-138. 
ISSN: 2574-1241

DOI: 10.26717/BJSTR.2019.21.003604

Hooshang Faghihi. Biomed J Sci \& Tech Res

(c) (i) This work is licensed under Creative BY Commons Attribution 4.0 License

Submission Link: https://biomedres.us/submit-manuscript.php

\begin{tabular}{ll} 
BIOMEDICAL & \multicolumn{1}{c}{ Assets of Publishing with us } \\
RESEARCHES & - Global archiving of articles \\
- Immediate, unrestricted online access \\
issN:2574-1241
\end{tabular}

